\title{
Prior Invasive Malignancy
}

National Cancer Institute

\section{Source}

National Cancer Institute. Prior Invasive Malignancy. NCI Thesaurus. Code C154619.

A finding indicating the presence of a previously diagnosed invasive malignant neoplasm. 